\title{
Prevención del maltrato infantil. Situación de la política pública en Bogotá D.C.*
}

\section{Preventing child abuse. The situation of public policy in Bogotá D.C.}

\section{Prevenção do maltrato infantil. A situação das políticas públicas em Bogotá D.C.}

Recibido: 26 de Julio de 2019. Aceptado: 27 de Enero de 2020.

Publicado: 1 de Octubre de 2020.

DOI: https://doi.org/10.11144/Javeriana.rgps19.pmis

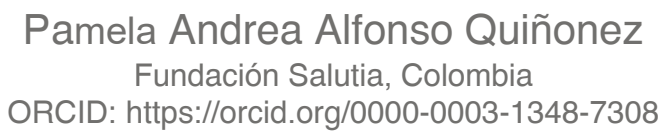

Ariel Emilio Cortés Martínez ${ }^{a}$

Pontificia Universidad Javeriana, Colombia ORCID: https://orcid.org/0000-0002-1169-9086

\section{Carmen Elisa Becerra Huertas}

Universidad Colegio Mayor de Cundinamarca, Colombia ORCID: https://orcid.org/0000-0002-4325-652X

Para citar este artículo Alfonso PA, Cortés AR, Becerra CE. Prevención del maltrato infantil. Situación de la política pública en Bogotá D.C. Rev Gerenc Polit Salud. 2020;19. https://doi.org/10.11144/ Javeriana.rgps19.pmis

a Autor de correspondencia. E-mail: ariel.cortes@javeriana.edu.co 


\section{Resumen}

Objetivo: comprender el fenómeno de la prevención del maltrato infantil entre los años 2008 y 2015 en la ciudad de Bogotá D.C., desde la perspectiva institucional y la normatividad vigente. Metodología: enfoque cualitativo de estudio de casos, con recolección, procesamiento y análisis de la información en diferentes organizaciones relacionadas con el maltrato infantil en Bogotá. Se utilizó la codificación desde el interaccionismo simbólico articulada en tres fases. Resultados: la política pública distrital de infancia y adolescencia tiene tres ejes para garantizar el ejercicio de los derechos de niños, niñas y adolescentes: garantías universales, superación de situaciones de limitación y restablecimiento de derechos. En cuanto a las percepciones de existencia de la normatividad, se encontraron problemas en la ejecución de la política pública y su planeación. Conclusiones: en la prevención del maltrato infantil a nivel distrital se observaron problemas en la ejecución de la política pública en el componente del trabajo intersectorial. Este abordaje depende de la identidad cultural y étnica, así como de personal capacitado.

Palabras clave: Violencia doméstica, maltrato a los niños, servicios de protección infantil, violencia, política pública.

\section{Abstract}

Objectives: To understand the phenomenon of the prevention of child abuse between 2008 and 2015 in the city of Bogotá D.C., from the perspective of institutions and current regulation. Methodology: A qualitative case-study approach was applied, including collection, processing, and analysis of information in different organizations related to child abuse in Bogotá. It was used the codification of symbolic interactionism, articulated in three phases. Results: The district public policy of childhood and adolescence has three axes to guarantee the exercise of rights by children and adolescents: universal guarantees, overcoming situations of limitation and restoration of rights. Regarding the perceptions of the existence of the regulation, problems were found in the execution of public policy and its planning. Conclusions: Prevention of child abuse at the district level shows problems in the execution of public policy in the intersectoral work component. This approach depends on cultural and ethnic identity, as well as personnel trained.

Keywords: Domestic violence, child abuse, child protective services, violence, public policy.

\section{Resumo}

Objetivos: Entender o fenômeno da prevenção do maltrato infantil entre 2008 e 2015 na cidade de Bogotá D.C., do ponto de vista institucional e dos regulamentos atuais. Metodologia: Abordagem qualitativa de estudo de casos, com coleta, processamento e análise de informações em diferentes organizações relacionadas ao abuso infantil em Bogotá. Foi utilizada a codificação do interacionismo simbólico articulado em três fases. Resultados: A política pública municipal da infância e adolescência tem três eixos para garantir o exercício dos direitos das crianças e adolescentes: garantias universais, superação de situações de limitação e restabelecimento de direitos. Com relação às percepções de existência da normatividade, foram encontrados problemas na execução de políticas públicas e seu planejamento. Conclusões: A prevenção do abuso infantil a nível municipal mostra problemas na execução de políticas públicas no componente das relações intersetoriais. Esta abordagem depende da identidade cultural e étnica, bem como de pessoal treinado.

Palavras-chave: Violência doméstica, maltrato infantil, serviços de proteção infantil, violência, políticas públicas. 


\section{Introducción}

Una de las primeras definiciones de maltrato fue la desarrollada por Henry Kempe (1962), que la ubicaba en el contexto de la violencia física (1). Para la legislación colombiana el maltrato es "toda forma de perjuicio, castigo, humillación o abuso físico o psicológico, descuido, omisión o trato negligente, malos tratos o explotación sexual, incluidos los actos sexuales abusivos y la violación y en general toda forma de violencia o agresión sobre el niño, la niña o el adolescente por parte de sus padres, representantes legales o cualquier otra persona" (2).

El Maltrato Infantil (MI) es un problema de interés "médico-social-legal que afecta al menor, la familia, y la sociedad" (3). Es una situación que ha aquejado al mundo desde los inicios de la civilización. En la antigüedad era aceptado ejercer acciones violentas sobre niños, mujeres y esclavos, y no era considerado un problema social (1). En la actualidad, en el marco internacional que sanciona el MI, la Convención de Derechos de los niños 1989, y los hallazgos clínicos, se ha demostrado cómo el MI afecta la funcionalidad de los individuos y de la sociedad. En la medida que este fenómeno social no ha podido ser controlado, se enmarca como un problema de salud pública (4).

Las consecuencias del MI se observan desde diferentes perspectivas. En lo individual, se plantea que se encuentran repercusiones sobre el desarrollo físico y socioemocional de los individuos sobre los cuales se ha ejercido la violencia las cuales pueden durar toda la vida (5). En lo social y laboral, la Organización Mundial de la Salud (OMS) refiere efectos negativos que influyen en el retraso del desarrollo de la sociedad (6).

Se sabe que además del control de los efectos posteriores al evento de MI, las estrategias de prevención permiten disminuir la probabilidad de que un evento ocurra, por lo cual se han planteado diversas estrategias (7). Algunos países las han incluido dentro de sus políticas públicas con programas dirigidos a fortalecer los vínculos familiares o acciones en escuelas saludables que han permitido tener un acercamiento a la prevención en salud del MI (8).

Existen tres tipos de prevención en salud, según el objetivo de las acciones o intervenciones. La prevención primaria comprende estrategias para impedir la aparición de nuevos casos de MI y se dirige a la población en general; la prevención secundaria se basa en estrategias dirigidas a impedir la aparición de nuevos casos en familias detectadas como de alto riesgo; y la prevención terciaria es la intervención cuando ya ha ocurrido el maltrato y se intenta impedir que ocurra nuevamente, al igual que palear las secuelas físicas y psíquicas en la víctima (9).

En Colombia han sido implementadas diferentes estrategias por parte del gobierno nacional, entidades territoriales y organismos no gubernamentales. Las políticas públicas dirigidas a la protección de la infancia han tomado relevancia en los planes de desarrollo, donde se analiza la situación de la niñez y la adolescencia, se identifica el problema y se priorizan las soluciones. 
En el caso de la ciudad de Bogotá D.C., investigaciones anteriores como la de Gómez et al. (10) y Barreto et. al. (11) han evidenciado la problemática del MI y sus consecuencias.

Por lo anterior, el objetivo de la presente investigación es analizar la situación de la prevención del MI entre los años 2008 y 2015 en la ciudad de Bogotá D.C. desde la perspectiva institucional y desde la normatividad vigente. Para ello surge la pregunta ¿Cómo es el fenómeno de la prevención del MI a nivel distrital?

\section{Metodología}

Se empleó un enfoque cualitativo de estudio de casos, el cual permitió la recolección, procesamiento y análisis de la información para comprender el fenómeno estudiado en diferentes organizaciones relacionadas con el MI ubicadas en la ciudad de Bogotá. La metodología utilizada fue la codificación desde el interaccionismo simbólico (12), articulada en tres fases.

En la primera fase se construyó una matriz en una hoja de cálculo (Excel) en donde se identificó una serie de ejes que se pueden identificar en las normas, entre las cuales estaban: el conocimiento de la norma, su implementación, factores que influyen en la implementación, fomento del ejercicio de derechos por niños, niñas y adolescentes y trabajo intersectorial en cuanto a la prevención del MI. A partir de esto se formularon las preguntas para la entrevista. Un ejemplo de pregunta para el eje de fomento del ejercicio de los derechos por niños, niñas y adolescentes fue: ¿cómo fomentan o promueven que los menores hagan un pleno uso de sus derechos? Se validó la entrevista semiestructurada mediante una prueba piloto con un experto temático.

En la segunda fase se escogieron cinco instituciones distritales y nacionales relacionadas con el MI, como son la Secretaría Distrital de Hábitat (SDH), el Ministerio de Salud y Protección Social (MSPS), la Dirección de Policía de Infancia y Adolescencia, el Instituto Colombiano de Bienestar Familiar (ICBF) y la Secretaría Distrital de Integración Social (SDIS); cada institución fue tomada como un caso independiente, con el fin de conocer las razones de coherencia entre las políticas nacionales y las estrategias implementadas. La información se recolectó mediante entrevistas individuales semiestructuradas con empleados públicos del nivel directivo de las cinco instituciones. La participación fue de carácter libre y con consentimiento informado. La entrevista fue grabada y transcrita garantizando el anonimato de esta.

En la tercera fase, la información recolectada se agrupo de acuerdo con los ejes planteados, realizando codificación abierta de carácter inductivo permitiendo conocer las percepciones de los entrevistados. Las codificaciones obtenidas se agruparon en categorías emergentes buscando relación entre éstas ${ }^{1}$. 


\section{Resultados}

La normatividad está regulada por la Constitución Política de Colombia en sus artículos 42 al 46, el Código de la infancia y la adolescencia expedido en la Ley 1098 de 2006, que contiene directrices claras frente a la protección de la infancia, y el Código Penal Colombiano, el cual especifica cuáles son las sentencias para los agresores.

Las entidades relacionadas con la protección de la infancia y la adolescencia se encuentran reguladas por el Sistema Nacional de Bienestar Familiar (SNBF). El objetivo primordial de dichas entidades y del sistema es "la protección integral de niños, niñas y adolescentes" (2). El SNBF otorga funciones de creación de programas y estrategias de prevención a las siguientes instituciones: Policía Nacional y dentro de la Policía de Infancia y Adolescencia, Instituto Colombiano de Bienestar Familiar (ICBF), MSPS, Ministerio de Educación (MEN), Ministerio de Trabajo, Ministerio de Vivienda y Comisaría de Familia. La responsabilidad de la política pública de infancia y adolescencia recae en la Presidencia de la República, Gobernadores y Alcaldes, el Departamento Nacional de Planeación (DNP), MSPS, ICBF y MEN.

El gobierno nacional expidió el Plan Nacional para la Niñez y la Adolescencia 2009-2019 (13), y el Plan Nacional de Construcción de Paz y Convivencia Familiar 2005-2015 (14). Estos incluyen acciones que buscan garantizar los derechos de niños, niñas y adolescentes, mejorar las relaciones y resolver los conflictos familiares para garantizar entornos saludables.

A nivel distrital se identificó el Decreto 121 de 2012, que crea el Consejo Consultivo Distrital y los Consejos Locales de niños, niñas y adolescentes, para brindarles la oportunidad de participar en las decisiones que puedan afectar su bienestar para garantizar el ejercicio de sus derechos $(15,16)$. El Acuerdo 12 de 1998, en el que se crea el Consejo Distrital de Atención Integral a los Menores en Abuso y Explotación Sexual (17). El Acuerdo 489 de 2012 por medio del cual se adopta el Plan de Desarrollo económico, social, ambiental y de obras públicas para Bogotá 2012-2016, que dentro de sus objetivos está "dar prioridad a la infancia y adolescencia con énfasis en la primera infancia y aplicando un enfoque diferencial en todas sus políticas" (18).

La política pública distrital de infancia y adolescencia tiene tres ejes para lograr el objetivo de garantizar el ejercicio de los derechos de niños, niñas y adolescentes en el Distrito: garantías universales, superación de situaciones de limitación y restablecimiento de derechos (16).

A continuación se exponen las percepciones de existencia sobre la prevención del MI a nivel distrital.

Conocimiento de la norma. Las entrevistas se enfocaron en conocer la profundidad del conocimiento de la norma aplicada al MI en especial en la prevención del MI, con lo cual se logró entender el adecuado conocimiento de estas por parte de las instituciones, y en especial las relacionadas con la protección y garantía de los derechos de niños, niñas y adolescentes. 
Todo lo que tiene que ver con brindarle a los niños y las niñas una garantía de sus derechos. Hay un artículo específico que habla del derecho a la salud de los niños y las niñas, en el cual el sector salud tiene que dar o brindar una respuesta específica, pero también en el marco del restablecimiento de derechos tiene unas tareas que cumplir cuando ya ha habido una vulneración, y hay unas tareas de prevención y promoción en temas la salud (P2).

Los entrevistados relacionaron sus funciones en el área de prevención como una estrategia para lograr garantizar la protección de la infancia. Lo que indica que conocen la norma existente, y plantean que la prevención no es función de una sola institución, sino de varias instituciones y se deben crear programas articulados para lograr prevenir el MI. "La prevención del MI tiene temas que involucran todos los sectores" (P2).

Implementación de la norma. Las entrevistas se direccionaron a conocer la planeación estratégica, en especial, en el componente de diseño estratégico traducido en programas y planes que cada institución viene desarrollando para la prevención del MI.

Los participantes dieron diferentes ejemplos de los programas y planes relacionadas con la prevención, entre ellos, el programa "De cero a siempre" del MSPS; "Abre tus ojos" de la Policía de infancia y adolescencia; "Yo protejo y promuevo entornos protectores y territorios seguros" de la SDIS. Los programas y planes implementados no son estáticos sino dinámicos lo cual implica ajustes en la gestión estratégica. A pesar de esto no son suficientes para lograr el objetivo para el cual fueron creados, ver tabla 1.

Tabla 1 Programas y/o estrategias de prevención de maltrato

\begin{tabular}{|l|l|}
\hline Institución & \multicolumn{1}{|c|}{ Programa } \\
\hline Ministerio de Vivienda & $\begin{array}{l}\text { Convivencia de arraigo territorial y participación en la } \\
\text { construcción social del hábitat. }\end{array}$ \\
\hline Ministerio de Salud & De cero a siempre. \\
\hline $\begin{array}{l}\text { Policía de infancia y } \\
\text { adolescencia }\end{array}$ & Abre tus ojos. \\
\hline ICBF & $\begin{array}{l}\text { Generaciones con bienestar, familias con bienestar, } \\
\text { Acciones Masivas de Alto impacto Social (AMAS). }\end{array}$ \\
\hline $\begin{array}{l}\text { Secretaria Distrital de } \\
\text { Integración Social }\end{array}$ & $\begin{array}{l}\text { Yo protejo y promuevo entornos protectores y territorios } \\
\text { seguros. }\end{array}$ \\
\hline
\end{tabular}

Fuente: elaboración propia.

La idea es poder llegar con diferentes opciones y con diferentes oportunidades novedosas a los niños y a las niñas, entonces aquí lo que tratamos de hacer es brindarles muchas oportunidades nuevas y brindarles opciones que a ellos les guste, que les llame la atención, y que ellos puedan participar activamente, se puedan expresar, decir qué les gusta, qué no les gusta y que su opinión sea tomada en cuenta, para que se construya con ellos lo que a ellos les gusta (P4). 
Se indagó sobre dichas oportunidades y nuevas opciones, profundizando sobre si estas estrategias pueden ser mejoradas, y se encontró que existen espacios para lograr perfeccionamientos en los mismos.

Pienso que sí estamos cortos en el escenario de la prevención. Acá activamos rutas cuando ya el delito se ha cometido. Hace mucha falta abordar mucho más a la familia, porque el fin último es una familia funcional que pueda, en medio de sus posibilidades, garantizar los derechos mínimos vitales del desarrollo integral de los niños (P5).

En cuanto a los efectos que han tenido con las estrategias, en general, todos consideran que han logrado, de una u otra forma, generar cambios en las personas y las comunidades con sus intervenciones a pesar de los resultados. Algunos de los impactos que han percibido con las estrategias de prevención están relacionados con cambios comportamentales, cambios a nivel familiar, autoconocimientos de los niños y el reconocimiento de situaciones en los que se pueden ver vulnerados. Todo lo anterior teniendo en cuenta un buen abordaje de las comunidades por parte de los empleados públicos.

Se ha logrado que los niños, las niñas y los adolescentes se empoderen de sus propios derechos (P4).

Factores que influyen en la implementación. Se buscó determinar las dificultades que influyen en la implementación de las políticas dirigidas a la prevención del MI, entre ellas se encontraron:

La falta de recurso humano capacitado y la alta rotación de este, lo cual entorpece la labor que se desarrolla en las comunidades.

Nosotros también manejamos unas herramientas para poder llegar a la población vulnerable de manera efectiva, con diferentes estrategias..., el cual maneja 18 de las 36 temáticas..., por falta de recursos (P3).

Las estrategias implementadas han tenido en cuenta las diferencias culturales y los diferentes tipos de maltrato. Los programas brindan la flexibilidad de incluir poblaciones indígenas y otras etnias, sin embargo, sigue siendo un reto. El enfoque que se le da a las acciones varía de acuerdo con la población.

Es difícil llegar a ellos, porque ellos dicen, ¡nosotros tenemos derechos!, los cuales son específicos para las comunidades indígenas (P3).

Eso depende del lugar a donde estemos llegando. Hay lugares en Colombia en donde es muy crítico el tema de reclutamiento de niños, niñas y adolescentes, por ejemplo, de lugares particulares donde se ven que los niños trabajan muchísimo (P1).

A pesar de esto, todos los entrevistados reconocieron que faltan estrategias y se limitan solamente a activar las rutas cuando el MI ya se ha cometido. 
Pienso que sí estamos cortos en el escenario de la prevención. Acá lo que hacemos es activar rutas cuando ya el delito se ha cometido, cuando ya está vulnerado el derecho, cuando los niños ya están afectados. Hace mucha falta abordar mucho más a la familia, porque el fin último es una familia funcional que pueda, en medio de sus posibilidades, garantizar los derechos mínimos vitales del desarrollo integral de los niños (P3).

Fomento del ejercicio de los derechos por niños, niñas y adolescentes. De igual manera, se buscó conocer en los entrevistados el fomento de los derechos.

Demostraron que las actividades que desarrollan buscan proteger los derechos de la infancia, pero no todos fomentan el ejercicio de sus derechos.

Hay un trabajo con grupos líderes, lo mismo para los temas de violencia de género..., y en general hay unas acciones para fortalecer esos procesos de participación de los niños y niñas, pero no nos corresponde ese ejercicio de la participación tan grande, pero sí nos hemos ido fortaleciendo para darles esa voz y ese espacio, como te digo, para que sean el centro de atención (P2).

Trabajo intersectorial. Las respuestas proporcionadas por los entrevistados dejaron ver que esto es algo que no funciona adecuadamente a nivel nacional y distrital probablemente por todos los actores institucionales que interactúan.

Las instituciones están haciendo todo lo posible por hacer las articulaciones. Entonces poco a poco cada institución va viendo cuáles son sus necesidades y, asimismo, se están encargando de actuar y buscan trabajar articuladamente (P3).

Algunos reconocen la importancia de que las estrategias de las instituciones no se limiten a atender los casos.

Prioridad como dice la ley, a su interés superior, y no centrados o moviéndonos solamente porque tuvo una vulneración y actuando cuando ya tuvo una vulneración, sino que siempre se debería actuar, y eso también viene con una mirada general de prevención (P2).

Resultado del análisis se identificaron, de un lado, como debilidades y amenazas: la falta de articulación y comunicación entre las instituciones y la constante rotación del personal. Y, de otro lado, como fortalezas y oportunidades: los programas existentes y el número de organizaciones relacionadas con el MI.

\section{Discusión}

Existe una conciencia creciente de que el MI es una amenaza endémica para la salud y la seguridad de los niños, tanto en América Latina (19) como en Colombia. Lo que conlleva a replantear la política pública. En cuanto a esta, el presente trabajo confirma lo planteado por Cunill-Grau (20), Lacruz (21) y la Comisión de Determinantes Sociales de la Salud (22), sobre la necesidad de invocar a la inter-sectorialidad como eje central de las nuevas políticas sociales 
en especial en el MI, la cual es una nueva promesa en materia de gestión para acometer objetivos de transformación social.

Coincidimos con Navarro (23), en que la política pública de prevención no se debería limitar a una política de atención en salud, sino que debería ser integradora y enfocada en reducir las desigualdades sociales, a generar cambios en los comportamientos y a empoderar a la comunidad, como lo plantea Cooper (24).

Se ratifica lo esbozado por Medina-López (25), la prevención del MI y de la violencia intrafamiliar se debe definir como una estrategia de coordinación interdisciplinaria, interinstitucional y comunitaria, que se constituya en un plan conjunto, dirigido a la prevención y atención de las diferentes formas de MI.

De igual manera, se encontraron obstáculos para poder implementar adecuadamente las políticas públicas dirigidas a la prevención del MI. Esto conlleva a que los resultados no sean buenos. Para lograr resultados es necesario tener un amplio entrenamiento de uso las rutas de servicios de conformidad a los hallazgos de Monteleone y Brodeur (7), y la presente investigación añade que es necesario no solo tener las rutas de servicios una vez ocurrido el MI, sino que se debe fortalecer la prevención del este a través de nuevas estrategias para evitar que el abuso ocurra.

Autores como Cuadros (9) manifiestan la importancia de tener estrategias dirigidas a abordar la prevención primaria, secundaria y terciaria, para lo cual es necesario tener un recurso humano calificado, esto último confirma nuestros hallazgos así como también lo manifestado por PérezRendón (26) y Paula et al. (27).

Se ratifica lo expresado por Oliván-Gonzalvo (28) acerca de que conocer las diferencias entre etnias de niños maltratados puede ser de utilidad para los profesionales de los servicios sociales comunitarios responsables de desarrollar estrategias de intervención para la prevención y la detección precoz del maltrato.

\section{Conclusiones}

En la prevención del maltrato infantil (MI), a nivel distrital, se observaron problemas en la ejecución de la política pública, en especial, en el componente de las relaciones intersectoriales y su abordaje. De acuerdo con los resultados de la presente investigación, esto se debe a que depende de la identidad cultural-étnica y falta de personal capacitado para lograrlo.

La normatividad no es específica frente a las acciones que se deben desarrollar para la prevención del MI, sin embargo, las instituciones han logrado crear programas, estrategias y acciones que buscan prevenir la ocurrencia de situaciones en las que los derechos de niños, niñas y adolescentes se vean vulnerados. 


\section{Agradecimientos}

Se agradece la participación a cada una de las organizaciones colaboradoras mencionadas en el presente artículo y a la estudiante de Nutrición y Dietética Laura González de la Facultad de Ciencias Básicas de la Pontificia Universidad Javeriana. Este artículo fue financiado por la Pontificia Universidad Javeriana en el marco del proyecto "Prevención del MI: situación de la política pública en Bogotá" que sirvió como trabajo de grado de la estudiante Pamela Alfonso. Los autores manifiestan no tener conflicto de interés y se hacen responsables de lo manifestado en el mismo.

\section{Referencias}

1. Ramírez C. El impacto del maltrato en los niños y las niñas en Colombia. Revista de Infancia y Adolescencia. 1994;1(2): 271-301. https://www.redalyc.org/pdf/769/76910207.pdf

2. Congreso de colombia. Ley 1098 de 2006. Por la cual se expide El Código de Infancia y Adolescencia; 2006.

3. Loredo A, Trejo J, García C, Portillo A, Capistrán A, Carballo R, et al. Maltrato infantil: Una acción interdisciplinaria e interinstitucional en México. Comisión Nacional para el estudio y la atención integral al niño maltratado. Salud Ment. 2010;33(3): 281-290. https://www.redalyc.org/pdf/582/582 16021009.pdf

4. Francia Reyes ME. Maltrato infantil: Un problema de todos. Rev Cuba Med Gen Integr. 2003;19(1). htt p://scielo.sld.cu/scielo.php?script=sci_arttext\&pid=S0864-21252003000100009

5. Fuster E, García F, Musitu G. Maltrato Infantil: Un modelo de intervención desde la perspectiva sistémica. Cad Consult Psicológica. 1988;73(4): 73-82. https://www.valencia.edu/garpe/C_/A_C_A_0003.pdf

6. Costa Cabanillas M, Morales González J. La prevención de la violencia en la infancia y la adolescencia: una aproximación conceptual integral. Psychosoc Interv. 2001;10(2): 221-240. http://www.copmad rid.org/webcopm/publicaciones/social/76940.pdf

7. Monteleone JA, Brodeur AE. Child Maltreatment: A Clinical Guide \& Reference. Child Maltreatment: A Clinical Guide \& Reference; 1994.

8. Butchart, A. Phinney A. Prevención del maltrato infantil: Qué hacer, y cómo obtener evidencias. Organ Mund la Salud; 2009.

9. Cuadros I. Manual básico para el diagnóstico y tratamiento del maltrato infantil. Save the children. 2005. Bogotá: Secretaría de Integración Social; 2005.

10. Gómez N, Granados N, Quintero D. Maltrato infantil en Bogotá, D.C., durante el período 2008-2009. Trabajo de grado. Bogotá: Editorial. Pontificia Universidad Javeriana; 2010.

11. Barreto Y, Enriquez C, Cordoba C, Rincon K, et al. Percepción de violencia desde escolares de dos instituciones educativas de la localidad de Kennedy, Bogotá. Rev Salud Pública. 2018;20(4):438-444. https://scielosp.org/pdf/rsap/2018.v20n4/438-444/es 
Prevención del maltrato infantil. Situación de la política pública en Bogotá D.C.

12. Strauss AL, Corbin JM. Basics of Qualitative Research: Grounded Theory Procedures and Techniques. London: Sage Publications; 1990.

13. Protección Social M de S. Plan Nacional para la Niñez y la Adolescencia 2009-2019.

14. Instituto Colombiano de Bienestar Familiar - ICBF. Plan Nacional de Construcción de Paz. Bogotá: ICBF; 2015.

15. Bogotá Alcaldía Mayor. Política pública de infancia y adolescencia. Decreto 121 de 2012. Bogotá: Alcaldía Mayor; 2012.

16. Alcaldía Mayor de Bogotá. Política de infancia y adolescencia en Bogotá D.C. 2011 - 2021. Bogotá: Alcaldía Mayor; 2011.

17. Alcaldía Mayor de Bogotá. Acuerdo 12 de 1998. Créase el Consejo Distrital de Atención Integral a los menores en abuso y explotación sexual como cuerpo consultor y asesor encargado de formular políticas y programas de las entidades responsables en Santa Fe de Bogotá, D.C. Bogotá: Alcaldía Mayor; 1998.

18. Alcaldía Mayor de Bogotá. Acuerdo 489 de 2012. Plan de desarrollo económico, social, ambiental y de obras públicas para Bogotá D.C. 2012-2016. Bogotá: Alcaldía Mayor; 2012.

19. Veenema TG, Thornton CP, Corley A. The public health crisis of child sexual abuse in low and middle income countries: An integrative review of the literature. International Journal of Nursing Studies; 2015.

20. Cunill-Grau N. La intersectorialidad en las nuevas políticas sociales. Un acercamiento analíticoconceptual. Gestión y Política Pública. 2014;23(1): 5-46. http://www.scielo.org.mx/pdf/gpp/v23n1/ v23n1a1.pdf

21. Lacruz T. Haciendo de la violencia familiar un asunto público, la experiencia en Québec, Canadá: Temas Coyunt. 2007;(55). http://revistasenlinea.saber.ucab.edu.ve/temas/index.php/temasdecoyuntura/artic le/view/1248

22. Organización Mundial de la Salud. Subsanar las desigualdades en una generación. Alcanzar la equidad sanitaria actuando sobre los determinantes sociales de la salud. Comisión sobre Determinantes Sociales de la Salud; 2009.

23. Navarro V. ¿Qué es una política nacional de salud ? CELA. 2018;(130): 39-58. http://biblioteca.clacso .edu.ar/Panama/cela/20120717092358/quees.pdf

24. Cooper A. A short psychosocial history of British Child abuse and protection: Case studies in problems of mourning in the public sphere. J Soc Work Pract. 2014;28(3): 271-285. https://doi.org/10.1080/ 02650533.2014.927842

25. Medina R. Manual de educacion preventiva sobre violencia intrafamiliar: la paz empieza por casa. Bogotá: ICBF; 1995. 90 p.

26. Pérez-Rendón JG, Flores-Pérez L, García-Piña C, Loredo-Abdalá A, et al. Consideración del problema de maltrato infantil en los planes de estudio de medicina y de especialidades médicas en México. Acta Pediatr Mex. 2015;36(2). http://www.scielo.org.mx/scielo.php?script=sci_arttext\&pid=S018623912015000200003 
27. Paula CS, Lauridsen-Ribeiro E, Wissow L, Bordin IAS, Evans-Lacko S. How to improve the mental health care of children and adolescents in Brazil: Actions needed in the public sector. Rev Bras Psiquiatr. 2012;34(3): 334-351. https://doi.org/10.1016/j.rbp.2012.04.001

28. Oliván-Gonzalvo G. Comparación entre los niños de etnia gitana e inmigrantes extranjeros ingresados en centros de protección por maltrato. Gac Sanit. 2004;18: 380-386. https://doi.org/10.1016/S0213 $-9111(04) 71848-8$

Notas

Artículo de investigación.

El proyecto de investigación fue aprobado por el programa de Maestría en Salud Pública interfacultades de la Pontificia Universidad Javeriana. 